\title{
Prospects for Scientific Visualization as an Educational Technology
}

\author{
Douglas N. Gordin and Roy D. Pea \\ School of Education and Social Policy \\ Northwestern University
}

\begin{abstract}
Scientific visualization has the potential to make science education more accessible and to provide a means for authentic scientific inquiry. The role of scientific visualization within science is explicated through examples of its use and by presenting a sociological account of science that portrays scientific visualization as an important new inscriptional system. Three examples of using scientific visualization within education are provided: the ChemViz Project at the National Center for Supercomputing Activities, the Image Processing for Teaching (IPT) project at the University of Arizona, and an undergraduate climatology course at the University of Chicago. Potential problems of integrating scientific visualization within secondary education are described, including students' need to learn basic scientific practices, incompatibilities between the format of the traditional high-school classroom and scientific inquiry, and the need for additional infrastructure within schools. Finally, possible solutions to these problems are described, culminating in a view of scientific visualization as a means to provide education with an important new inscriptional system for exploratory, inquiry-driven learning and to link the aims of education to the practices of science.
\end{abstract}

Scientific visualization (SciV) stands for diverse scientific and social enterprises that include a new type of graphic representation; the creation of dramatic scientific images and their animation; an emerging academic field that combines elements of science, computing, semiotics, and the visual arts; and the coordination of a suite of advanced technologies to collect, store, process, and image large data sets. The rallying cry of SciV has acted as a catalyst to generate new lines of scientific inquiry and to push beyond the

Requests for reprints should be sent to Douglas N. Gordin, School of Education and Social Policy, 2115 North Campus Drive, Northwestern University, Evanston, IL 60208. 
frontiers of computer graphics to produce new methods of representation. In this article, we consider how to place the considerable momentum of SciV at the service of science education.

There are compelling reasons to bring SciV into education. Scientists increasingly rely on the techniques of SciV in pursuing their lines of inquiry and in communicating results to the scientific community. For example, within medicine, three-dimensional reconstructions of the human body are being formed from magnetic resonance images; atmospheric science has developed movies showing the development of tornadoes and the emergence of a hole in the ozone layer of the Earth's atmosphere; physicists are portraying the subatomic microstructure of material; and astronomers are animating the processes presumed to underlie the emergence of galaxies and black holes. These techniques have been widely accepted by scientists and are already fundamental to the production of scientific results in widely varying areas of concentration. Numerous scientific meetings on SciV now take place each year. The scientific community's increasing use of SciV in itself compels the attention of the education community because a major function of education is to prepare students with the skills and tools that professional fields employ. In addition, the compelling visual attraction of these methods and their ability to provide concrete images for abstract conceptions that are often portrayed clumsily in textual or diagrammatic descriptions also attract educators because SciV may be a valuable ally in the attempt to demonstrate the connection of science to its phenomena.

These incentives are challenged by the difficulties of transferring the methodologies of science and the technologies of the broader community into the classroom. Past crashes of optimistic expectations in this area are well documented (Cohen, 1988; Cuban, 1986; Griffin \& Cole, 1987). Using these analyses as sobering lessons has broadened our perspective. In particular, we acknowledge the situated nature of the tools and methodologies that we wish to introduce to the classroom. Hence, SciV cannot be extracted from the fields that have defined it and deposited, like a load of freshly minted textbooks on the doorstep of the schoolhouse. Instead, scientists and science educators must lead students to acquire the practices of the tools they use to pursue their craft of finding and forging descriptive patterns that reveal (or can come to signify) order amidst the seeming chaos of phenomena.

Our thesis is that SciV has the potential to make science education more accessible and to provide a means for authentic scientific inquiry. This is argued by presenting SciV as it is used within the scientific community and then describing how SciV can aid learning. Next, we describe the role of SciV within the science community through examples of its use and through a sociological account of science that emphasizes the role of inscriptions in the production and negotiation of knowledge. In the section entitled SciV Within Education, we present the potential of SciV for education by enumerating the ways that SciV can aid learning and by describing three examples of its use within educational settings - namely, the ChemViz Project, the 
Image Processing for Teaching (IPT) project, and an undergraduate climatology course at the University of Chicago. In addition, potential problems in using SciV within education are enumerated along with suggestions for their solution.

\section{ScIV}

This section presents SciV within science by considering it from several points of view that range from substantive to sociological. First, representational characteristics of SciV are examined in order to provide a rough overview of its distinctive characteristics. Second, an example is provided that uses SciVs to explore basic patterns of climate, thus demonstrating how SciVs can be used to view and reason about fundamental scientific forces. These two views are intended to provide a substantive view of $\mathrm{SciV}$, thus detailing how a scientist may describe SciV and its utility for reasoning about the world. Third, the practices surrounding the use of SciV within the scientific community are described, especially the standardization and distribution of large data sets. Fourth, a sociological perspective is adopted that views SciVs as inscriptions that scientists use to produce knowledge, to persuade their colleagues, and compete for scarce resources. These latter views show SciV within a broad social context. In following sections, these varying views will be used to provide the basis for understanding how SciV can be integrated with education, including its potential benefits and problems.

\section{Description of SciV}

The field of SciV is newly formed and thus resists clear definition. It is as much a social construction as a cleanly defined area of inquiry. A landmark report from the National Science Foundation (NSF; McCormick, DeFanti, \& Brown, 1987) established the field of SciV by linking disparate elements from the disciplines of science, computer science, and the visual arts. Because the defining characteristics of this emerging area are still being sought, the images it has produced still provide the most compelling description of this new discipline. Overall, the significance of these images is highly specific to the domains that produced them because they portray the phenomena studied by each scientific field. However, they can be distinguished by the following characteristics:

1. Usually incorporate massive amounts of data.

2. Aim for verisimilitude with the phenomena they represent.

3. Aim to represent entire phenomena represented holistically by interpolating from data. 
4. Use color extensively to encode the magnitude of variables.

5. Animate sequences to show the progression over time.

6. Rely on high-speed computation (e.g., supercomputers).

7. Usually represent spatial phenomena (Becker \& Cleveland, 1991).

The basic methodology of SciV can be explained through an extended comparison with digital photography. A digital photograph is made up of many pieces, called pixels, each of which has a shade. The values of these pixels are determined when the photograph is shot so that the more light received at a particular area, the higher the value recorded. When the digital photograph is graphically rendered, each pixel is mapped to a shade of gray indicated by these numbers. A SciV image is similar to these digital photographs in that it too is stored as a set of values that can be rendered by mapping each number to a particular shade. However, the values composing a SciV image are typically not formed from the intensities of visible light; rather, the numbers may represent abstract qualities. An example data set is global surface temperature (see Figure 1).

These temperatures are then viewed as a digital image in which each number is mapped to a specific shade. This "temperature portrait" allows one to discern patterns and contrasts much as figure and ground are seen in a photograph (e.g., in Figure 1, the high desert temperature of Australia's outback shows up as red against the yellows and greens of the cooler coastal land and ocean). As seen in the figures, the values need not be mapped to shades of gray. In contrast, the extensive use of color is a hallmark of SciV. Colorful palettes such as a full rainbow of colors or the color spectrum of molten metals can be used. Different palettes serve to bring out distinctive features of an image, thus encouraging an association with the phenomena under study. SciV also uses video to show a sequence of images that provides the appearance of change over time; for example, the annual temperature cycle could be illustrated by animating a sequence of SciVs that shows daily temperature over the course of a year. Another way of showing change over time is through three-dimensional renderings. Pixels are rendered as cubes (or voxels) instead of squares, and multiple data sets are piled on top of one another (Elvins, 1992). This three-dimensional space can then be explored by reducing it to selected slices or planes or rendering some set of values transparent, allowing one to peer into the internals of the assembled data. A crucial point is that the visualization algorithms usually attempt to represent spatial phenomena from a sampled set of data points. Thus, the resulting visualization portrays the original phenomena by making local interpolations. In contrast, graphs often attempt to find a mathematical formula that characterizes a set of data points. This formal simplicity is not required in SciV because our visual perceptions are well suited to discriminating complex patterns and anomalies. The previous description provides a relatively complete account of how SciV uses raster or pixel based images to render data sets. 
Other representations, including contour lines and arrows (Brodie et al., 1992), are used. Contour lines are placed around areas that have roughly the same values. The most common uses of contour lines are in topographic maps to show elevation. The rate of change can by observed from the spacing of lines: When lines are bunched together the rate of change is higher than widely separated lines. Contour lines can be appropriately used on many types of data; for example, a contour map of temperature can be used to indicate temperature values and rates of change.

Arrows are used differently than contour lines or raster images in that each arrow encodes two values-typically a direction and a magnitude. Arrows are commonly used for mapping winds, indicating the wind's direction by the arrow's angle and the wind's strength by the length of the arrow. However, any data set made up of pairs can use arrows. For example, the stress on an airplane's wing is often shown using arrows. The previous description of visual representations surveys some of the representations commonly used for SciVs of climate, which is the domain of the next example. Other scientific domains, such as biology and chemistry, rely much more extensively on three-dimensional modeling. However, a description of these methods or a full survey of representations used in SciV is beyond the scope of this article.

\section{Example of SciV}

In order to go beyond the abstract description just provided, we now examine some SciVs to show how they can be used to understand scientific phenomena. These examples continue to look at the temperature of the earth through presenting factors that underlie the range and distribution of the surface temperature. Consider Figure 2 (data from Barkstrom, 1984), which shows a monthly mean of the insolation (i.e., energy coming to Earth from the sun) during January 1987.

This is an unusually simple SciV because each horizontal line has a constant value. Hence, it could also be rendered as a two-dimensional graph. However, there are benefits to presenting the insolation as a colorful SciV. Showing the horizontal lines accentuates that there are spatial regions (in this case, along lines of latitude) that are receiving the same amount of energy or radiation. The colors dramatically encode the energy levels, ranging from the hot reds in the Southern Hemisphere to the chilly blues and purples in the Northern Hemisphere. In beginning to explore this SciV, certain familiar places on the globe can be examined for their value. For students in North America, a natural place to look first is where they live. Look at the thick band of blue covering North America-these chilly blues present a sharp indication of winter's cold. This can be used to demonstrate the causal relation between sunlight and our temperature, namely that it is 
sunlight that warms our planet. However, other examples are not as clear. Find the equator at $0^{\circ}$ latitude, which shows up as a bright yellow, thus revealing a high value but by no means the highest. This provokes intriguing questions: If the equator is not getting the most sunlight, why is it generally considered the hottest place, and if the South Pole is receiving so much solar energy, why is it so cold? To try to answer these questions, look at another SciV of insolation--this one from July 1987 (Figure 3; data from Barkstrom, 1984).

The SciV of July's insolation provides a seemingly flipped image from January's insolation. Here (in Figure 3), the North American summer is consistent with the red band crossing our continent. The earlier questions can now be partially answered, because in this SciV the equator is still a bright yellow, whereas the South Pole is a dark purple. Hence, a partial explanation for higher temperatures at the equator is that relatively high amounts of solar energy are received there throughout the year, whereas the amount received by the poles varies widely between seasons.

Now we may examine a SciV of the Earth's albedo (i.e., the reflectivity of the Earth's atmosphere system) for January 1987 (Figure 4; data from Barkstrom, 1984) to obtain additional insight into the varying patterns of surface temperature we are attempting to understand. Note that this SciV uses a different palette than the rainbow palette of the preceding SciVs. Here the palette varies from dark blue to white to dark red. The values of albedo can range from 0 to 100 , where 100 means that $100 \%$ of the sun's energy would be reflected back to outer space and 0 means that none of the sun's energy would be reflected, rather it would all be absorbed. Find the continents of South America and Africa in Figure 4, which show substantial areas of white in contrast to the dark blue of the surrounding oceans, thus indicating the continental land masses reflect more energy than their surrounding oceans. The high albedo (shown as bright red in Figure 4) of the South Pole reveals another reason for its extreme cold-most of the sun's energy is reflected away from it by polar ice. In contrast, a wide blue swath surrounds the equator signifying its low albedo and the fact that it will absorb most of the sun's energy that comes to it.

The complexity of the albedo also provides one reason why the temperature patterns on Earth are not as simple as the straight lines of insolation that Figures 2 and 3 indicate. Temperature patterns come from a combination of the influences of insolation and albedo as can be demonstrated using Figure 1, which shows a SciV of temperature for January 1987. Compare the bands of color denoting temperature in the Northern Hemisphere in Figure 1 with the bands of color denoting amount of incoming sunlight (or more technically, amount of incoming energy or insolation) in Figure 2. There is a parallel between the two, in that bands of purple, blue, and yellow can be roughly seen in both, running from the North Pole to the equator. The pattern in the Southern Hemisphere is more complex. Whereas the insolation shows a band of yellow followed by a large band of red, the temperature shows 
successive bands of yellow, green, and blue. Examine the albedo in Figure 4 to see why high temperatures, signified in Figure 1 by red, do not show up uniformly in the Southern Hemisphere. Around the equator, there are high temperatures because the low albedo (shown as blue in Figure 4) lets in (i.e., does not reflect) the insolation (shown as yellow in Figure 2); however, the higher values of the albedo (shown as white in Figure 4) in the middle of the Southern Hemisphere reflect the insolation, causing the temperatures to sink to a middling value (shown as green in Figure 1). The high albedo of Antarctica (shown as deep red in Figure 4) reflects almost all of the sun's energy, resulting in very cold temperatures (shown as blue and purple in Figure 1).

The seasonal change in temperature (see Figure 5) provides another illustration of how SciVs can be used to explore phenomena. Whereas the earlier examples derived insights by flipping back and forth between SciVs and finding causal patterns and connections, this SciV represents a derivation. Hence, the data in it were never directly observed; rather, they were calculated by subtracting the surface temperature of January 1987 from that of July 1987. A prominent feature of this seasonal difference SciV is the predominance of color (both red and blue) on the continents while the oceans are either white or very pale. Looking at the scale, white signifies a small change in temperature, whereas the darker blue and red hues signify large changes. Hence, this SciV demonstrates that much larger changes in surface temperatures occur on land masses than on the oceans. Such an observation could give rise to a line of inquiry to understand its cause. ${ }^{1}$

As presented earlier, SciVs provide the ability to pursue scientific inquiry through reasoning qualitatively about pictorial patterns. In the previous example, swaths of color were used to reason about the distribution of temperature on Earth. This style of reasoning utilizes patterns and color to perform a qualitative analysis of basic scientific phenomena. Finding these patterns relies on having particular knowledge about the world and the way that data sets represent it. Thus, rather than presenting an understanding, the SciVs form the basis around which an understanding can be woven. This is similar to the way a path can be constructed using a map. The map does not supply the path, rather the map provides an analogical space in which the referents of the origin and destination can be connected through a constructed path. Like maps, SciVs are not territory but are symbolic constructions with a purposive or intentional context. Which aspects of phenomena we choose to place on these representations and how we place them tell as

\footnotetext{
'The temperature of land masses varies more directly with absorbed insolation than does the temperature of oceans because of two primary factors. First, the surface of the oceans exchange heat with their underlying water at a greater rate than the surface of land exchanges heat with the underlying earth. Second, the specific heat of water (i.e., the amount of energy required to change its temperature) is greater than that of land; hence, when exposed to equivalent amounts of energy, its temperature changes more slowly.
} 
much about us as the SciVs tell about the physical reality from which they were abstracted. Yet, once constructed, SciVs can provide a powerful vantage point from which to make observations on the "constructed world" shown in them. In addition, these observations can give rise to questions or hypotheses about the world far more readily than can be obtained by reading arrays of numerical values or textual descriptions. By manipulating the underlying data sets, these hypotheses can be tested and verified. In this way, SciVs can help to integrate the too-often separated areas of model design and model testing.

\section{SciV Within the Scientific Community}

A description of SciV must include its contexts of use. These contexts are helpful in understanding the uses to which SciV has been put and what material and social requirements exist for its appropriation. These considerations are especially important when we come to consider how science education can appropriate SciV. Next, we briefly consider the fields of climatology and biology. Particular attention is drawn to the accumulation and standardization of data sets to facilitate the usage of SciV within these fields. The promulgation of these standardized data sets and SciVs derived from them have allowed scientists to distribute their knowledge and practices. These developments can be considered to be part of a continual and ubiquitous reification of knowledge into tools, which has been called the creation of "distributed intelligence" (Pea, 1993b).

Within the scientific community, the search for patterns within SciVs has been aided by the complementary practices of creating large standardized data sets that are used to construct SciVs and to provide the bases for experiments. Examples of these paired practices of searching for patterns and accumulating standardized data sets can be found in several scientific fields. Climatologists have built impressive repositories of data in recent years that are investigated through the coupled usage of SciV and Global Circulation Models (GCMs). Data sets are compiled and certified at national laboratories like the National Meteorological Center, the Carbon Dioxide Information Analysis Center, and the European Center for Medium Range Weather Forecasting, and then sent to researchers over high-speed computer networks or sent as CD-ROMs (Domenico, Bates, \& Fulker, 1994). Much of the experimentation performed with this data uses GCMs to understand the climate of the past (paleoclimatology) and to predict the climate of the future (Hall, 1992). These experiments function by seeding the GCMs with observed data and then iteratively applying differential equations defined by the model to predict the future climate. Often, these models are run through hundreds or thousands of iterations to predict the Earth's climate hundreds and thousands of years into the future. The end results are displayed as SciVs 
so that scientists can look for patterns of change in the amount and distribution of temperature.

Analogous practices are used within the biology community to map out the genetic codes that determine the development of life. Substantial progress has been made in mapping out the entire human genome. SciV has been an integral part of the history of understanding the structure of DNA as is recounted in Watson's (1968) classic account of discovering that DNA is structured as a double helix. Whereas Watson and Crick's original model was built from wire, more recent SciVs of DNA are generated on computer screens. The discovery and visualization of the structure of DNA has provided a basic foundation for later work in genetics and biochemistry, such as helping to build maps of chromosomes, thus leading to the localization of genes responsible for diseases. For example, the gene responsible for Hutchinson's disease has been localized, enabling researchers to test for its presence and offering crucial information to couples planning to have children. Thus, the basic practices of establishing standardized data sets for experimentation and understanding these data sets through SciV are present in biology as they are in climatology. Similar descriptions could be constructed of analogous practices in many other scientific fields such as medicine, chemistry, astronomy, and meteorology (for an excellent collection of SciVs from a wide variety of fields, see Wolff \& Yaeger, 1993).

The representations and tools utilized by a field often have a pervasive effect on what issues are examined and what problems are explored (Toulmin, 1953). This has often been overlooked within science, wherein scientists have argued that their representations are mirrors of nature or that they are deductively inferred from nature. Yet, as Toulmin persuasively argued, the relation between representations and phenomena are not deductive; rather, the representations provide a model complete with symbols that can be formally manipulated so that points of connection can be forged from the model to the phenomena. The strength of a model lies in its ability to encourage fruitful analogies to the phenomena to which it has been connected, thus leading to novel explorations. These models can also be considered to be systems in which knowledge is produced or manufactured. This point of view has been elaborated by sociologists of science.

\section{SciV as an Inscriptional Medium}

In recent years, sociologists have provided a startlingly different account of what scientists do when they "do" science, the roles which the external representations they produce play in that process, and a characterization of why various representations are successful in scientific discourse (e.g., Knorr-Cetina, 1981; Latour, 1988; Latour \& Woolgar, 1986; Lynch \& Woolgar, 1988). Because this account helps explicate the culture of science and its use of SciV, we see it as useful for understanding how to bridge the 
practices of high-school classrooms with those of scientists' laboratories and how the students may benefit from using SciV. Two methodological assumptions underlie much of this work and serve to illuminate why it differs from many conventional accounts of scientific practice. First, sociologists of science engage in a practice of anthropological strangeness. This refers to a conscious suspension of preconceptions and knowledge about the role of scientists in society, the meaning and purpose of the representations that scientists use, and the epistemological status of the products of science. Second, sociologists assume that the cognitive and perceptual mechanisms practiced by people are roughly the same independent of both culture and time. Hence, the abilities of scientists do not rely on uncommon insight or a qualitative jump in intellect, but utilize creativity and perceptions in an analogous way to that shown by Aborigines hunting for game, Bedouins navigating the deserts, and high-school students angling for a top grade.

In this characterization of science developed from empirical studies in diverse fields (e.g., Lynch \& Woolgar, 1988), the various formulas, diagrams, pictures, and textual accounts have been collectively labeled inscriptions to emphasize their nature as materially embodied representations. These inscriptions play a pivotal role in the actions of scientists. We may only briefly review these claims and their implications here. Vast amounts of work go into the production of inscriptions; the preparation for one inscription uses others, and debates over issues raised in these inscriptions dominate routine conversations in scientific workplaces. Science is thereby defined in these studies as the creation of these inscriptions and the social practices that accompany their creation and use. This perspective does not deny that science is about providing powerful explanatory models of the phenomena of the physical world, but it seeks to highlight the dominant character of inscriptions in scientific practice. In particular, two characteristics of inscriptions describe their use and success in the scientific enterprise. First, these inscriptions are used agonistically in argumentation as persuasive devices that can be used to compel agreement among peers. For example, a conversation could center on the quickest way to traverse a maze. To demonstrate a solution, a map of the maze is drawn with different paths shown in different colors, and the lengths of the paths are annotated. This inscription then becomes a powerful tool for resolving the debate. The victors of these arguments are rewarded socially and economically. Winning a scientific debate often determines the bottom line on societal awards such as grants, scientific stature, academic tenure, and influence.

Second, various formal and material aspects of inscriptions imbue them with power. These attributes of inscriptions are enumerated as immutable, mobile, flat, scalable, reproducible, recombinable, superimposable, able to be placed in written texts, and mergeable with geometry (Latour, 1988). Although we do not review accounts and definitions for these attributes here, these qualities can be seen in the map of the maze previously discussed when it is drawn on paper. Indeed, the enormous utility of paper for achieving 
these goals becomes an important factor in the success of inscriptions. Thus, paper becomes a common medium that permits various inscriptions to be combined, referred to, and accumulated. This reconstruction of science by sociologists of science continues on to the epistemological status of inscriptions. Whereas scientists frequently ascribe a basis in nature for their inscriptions, this sociological account finds them to be constructed in practice through a layering of representations. Using the previous maze example, the specialized map that is constructed builds on accepted representations of maps for describing spatial relationships and on representations in mathematics for summing distances. This example underscores the observation that representations are overlaid and built on after they have become widely accepted. In addition, an effective inscription often relies on resemblances with the object it represents. These resemblances are crafted, and as such, their use may undergo substantial development as was the case with realistic painting. The view of scientific material thus changes from a reflection of nature, as some theories of science would have it, to the products of craft or even to a manufacturing process (e.g., see case studies in Lynch \& Woolgar, 1988).

Using the previously mentioned framework, SciV can be considered as another inscriptional mechanism, thus providing for a more nuanced perspective on the role of SciV in science than the one we described earlier. Specifically, the following points can be made. First, SciVs can be described using the attributes of inscriptions, namely immutable, mobile, scalable, reproducible, and recombinable. These attributes are arguably all present, though with the computer, rather than paper, serving as the integrating environment. The movement of central locus from paper inscription to computer inscription has been widely anticipated (as in "the paperless office," although it is rarely found), but has occurred in the domain of SciV due to the reliance on high-quality color images which are difficult to render on paper and because of the extensive use of animation, which requires dynamic media such as computer or video displays. The loss of paper's flexibility is more than ameliorated by the seemingly endless ability of computers to store, present, and rearrange an increasingly wide variety of inscriptional forms in a rapid manner.

Second, SciV is used to garner consensus in specific substantive controversies and to obtain economic and other forms of backing for scientists in general. This perspective is well exemplified by SciV, as its creation as a field of study is linked to a report on the successful establishment of billions of dollars in government funding (Office of Science and Technology Policy, 1991), and SciV is being touted by Vice President Albert Gore as an important part of high-tech policy for the United States as part of the National Competitiveness Act (1993). Furthermore, there are many examples of SciVs being used to garner consensus on major scientific issues such as visualizations of the ozone layer to summarize and dramatize the debate over chlorofluorocarbons (CFCs) altering our atmosphere, medical sonograms of 
fetuses to emphasize their human attributes to dispute the legality of abortion, Landsat satellite images of floods and forest fires to illustrate the extent of disasters, images showing predictions of the Earth's warming to argue for reducing carbon dioxide emission, and animations of thunderstorms and other weather phenomena to predict and describe weather.

The aforementioned sociological portrait of science highlights the role of inscriptions within the practice of science. In this framework, the activity of science can be described as the production of inscriptions and of argumentation around those inscriptions. As an increasingly ubiquitous new inscriptional mechanism, SciV plays an important part in the way science is practiced. In particular, SciV provides a new medium in which scientists can observe patterns that are taken to represent the natural world. Alternatively, SciV provides a new medium in which patterns can be crafted to depict nature, thus forming the basis for analyses and descriptions of the natural world. In short, SciVs provide a new plastic medium in which to craft the inscriptions that constitute a primary activity in the practice of science. Similar to any representational system, SciV can be said to promote or "afford" certain modes of inquiry more than others. The animated and colorful images of SciV promote the use of pictorial patterns. The colorful representations like those in Figures 1 through 5, afford visual inferences and reasoning through pattern differences and cross-figure relations due to human perceptual discriminative capacities. Once again, it is not enough to say that the patterns are found-just as often they are crafted.

This essential tension has also been described with respect to our visual perception through two competing rules of thumb: we see what we expect to see versus our attention is caught by unusual features (Szlichcinski, 1979). Though seemingly contradictory, these complementary practices are basic to perceptual experience and are commonly practiced in the temporal sequence of work patterns utilizing SciVs. First, the choice of what data to examine and the way in which they are examined is determined by preconceptions that there is something interesting to be seen. Yet, given an open mind in a subsequent search for patterns, new possibilities can intrude. An interesting case in point comes from recent scientific history of how the ozone hole ${ }^{2}$ was detected (Hall, 1992).

In brief, the story is that satellite data detailing a precipitous decline in ozone were collected for several years without anyone taking note of their importance. The problem was that during data analysis, any measurements falling outside of "reasonable limits" were thrown out. Only after a different research team reported a drop in ozone levels as determined from simpler ground instruments, were the data reexamined and found to show a dramatic drop in ozone during the Antarctic spring months, roughly from August to

\footnotetext{
${ }^{2}$ Properly speaking, there is not a hole in the ozone; rather a thinning of the ozone layer has occurred above Antarctica.
} 
November. Despite the failure to use satellite data in discovering the problem, it subsequently has served as the basis for many dramatic SciVs depicting the grave condition of the earth's ozone shield. Arguably, these images, as much as any scientific arguments, helped consolidate the international communities' agreement to legislate the use of CFCs in an effort to reverse the depletion of the ozone layer.

\section{SCIV WITHIN EDUCATION}

\section{Roles for SciV Within Education}

The previous discussion described SciV and its role within science, thus setting the stage for a consideration of its potential within education. Ways in which SciV can aid science education include the following:

1. Making a scientific view of the world more accessible.

2. Providing a means for authentic scientific inquiry.

3. Empowering students with tools they can use in a wide variety of fields.

4. Laying groundwork to enable students to understand and critique scientific policy.

\section{Making Science Accessible}

Often, science education has consisted of memorizing formulas and learning to apply them using algorithmic processes (Linn, diSessa, Pea, \& Songer, in press; Linn, Songer, \& Eylon, in press). These abstractions contrast sharply with the complexity and beauty of the physical world that is the subject of science. SciVs can provide a more accessible inscriptional system for students to understand the subject matter, processes, and results of science. Through the use of color and animation, SciVs aim at achieving verisimilitude with the phenomena they represent. These correspondences, unlike mathematical formulas, rely on pictorial similarities that students can often readily assimilate. The dynamic time-based processes that science studies can be shown dynamically as animations that move through time, thus providing a more accessible means for reasoning about scientific processes than typical graphs, formulas, and quantitative analyses. For example, an animation showing monthly mean temperatures for a year (similar to Figure 1) could provide a basis for investigating and understanding patterns of seasonal change. This style of reasoning attempts to describe basic scientific principles qualitatively by referring to patterns of color in the SciV. For example, the role of albedo in redirecting sunlight can be discussed using Figure 4 , as in the example just mentioned. We do not imply that making the 
SciVs available will allow students to immediately understand the lines of inferences presented. Rather, these SciVs provide visible referents for the scientific processes that science education encourages them to appropriate and use as anchors in their inquiries.

This use of pictures and animations may also correspond to the strengths of "the Nintendo (or Sega) generation," so designated because of children's increased use of video games. The emergence of a more visual culture has been derided by educators seeking to foster written literacy (Postman, 1992), yet it can also play to the advantage of education through the use of engaging and intellectually stimulating images, such as SciVs.

\section{Providing the Means for Authentic Scientific Inquiry}

As already mentioned, teaching science through abstract formulas, schematized methodologies, and rote procedures has been the norm in our highschool and college classrooms. Yet, this neither captures what scientists do, nor is it successful in helping students appropriate the fundamental practices and concepts of science. In reaction to this failure, a new pedagogy has been articulated that strives to connect the practices of scientists and students. This new pedagogy, cognitive apprenticeship (Brown, Collins, \& Duguid, 1989; Pea, 1992), seeks to reemploy the practices of apprenticeship to provide education in areas not commonly associated with skills or trade, such as mathematics and science. A similar reconceptualization has emphasized education as enculturation into a community of practice in which students are peripheral members of the community (Hawkins \& Pea, 1987; Lave \& Wenger, 1991). As such, it is important that students observe how full members of the communities perform their tasks and that they have opportunities to use similar tools and inscriptional systems. Inquiry using SciVs can link students with the authentic practice of scientists. By utilizing the same SciV techniques and data sets as scientists, students gain a common ground with them that can promote mentor-to-apprentice relationships. Furthermore, the SciVs and their data sets provide the means for students to engage in authentic inquiry on basic scientific questions. Often students' experiments have had only a distant relation to the phenomenon under study (e.g., studying a tornado by swirling water in an aquarium). Though this type of experimentation can be valuable, SciVs allow students to interact with current scientific data collected from actual phenomena and ask their questions in relation to it.

Significant increases in student motivation can also result when students have the opportunity to study scientific questions that can have important ramifications on their lives. For example, many students are aware of a problem with the amount of ozone in the atmosphere. Although students may not understand the details behind the loss of ozone or why the thinning of ozone has occurred over Antarctica, they are aware that it poses a potential 

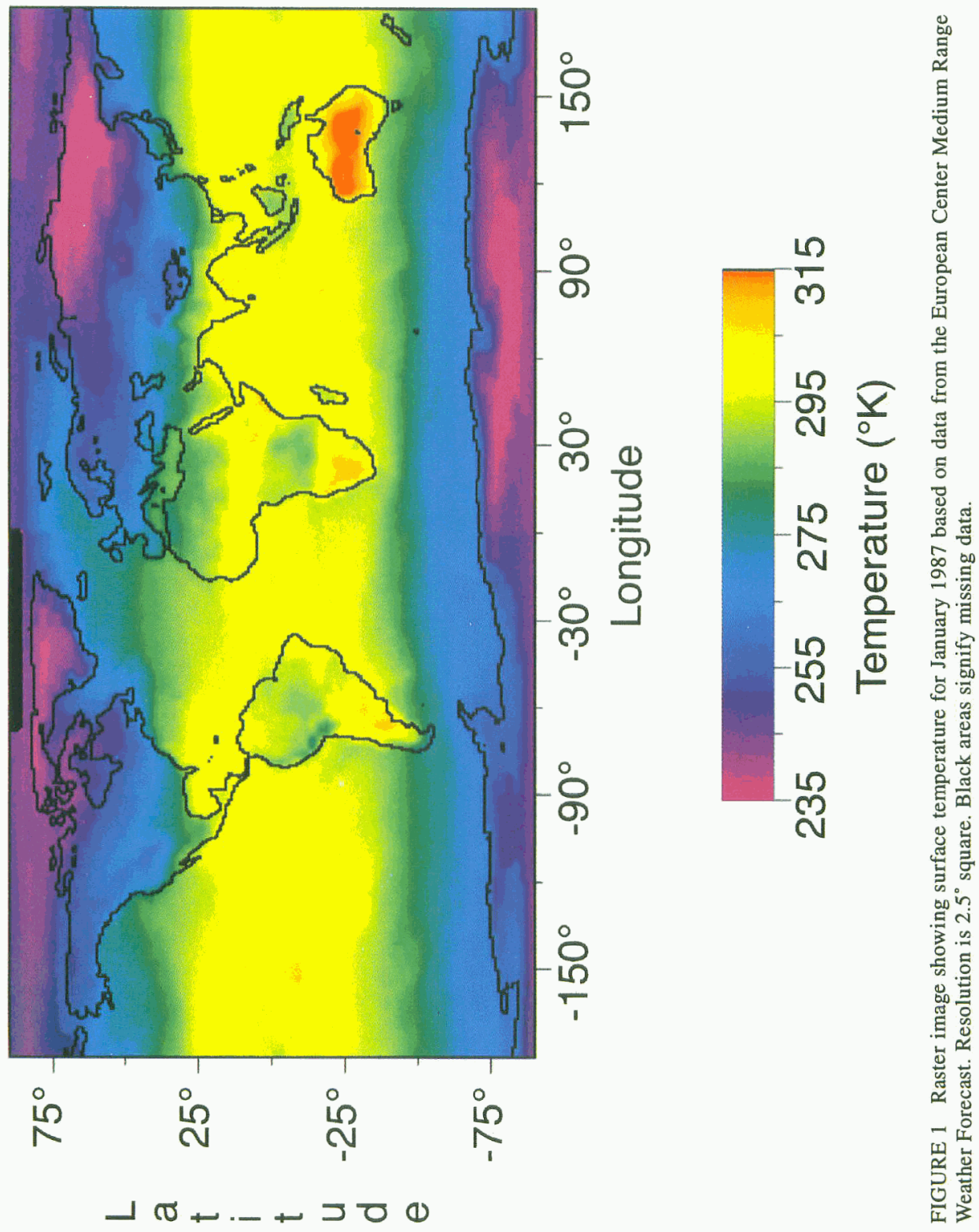

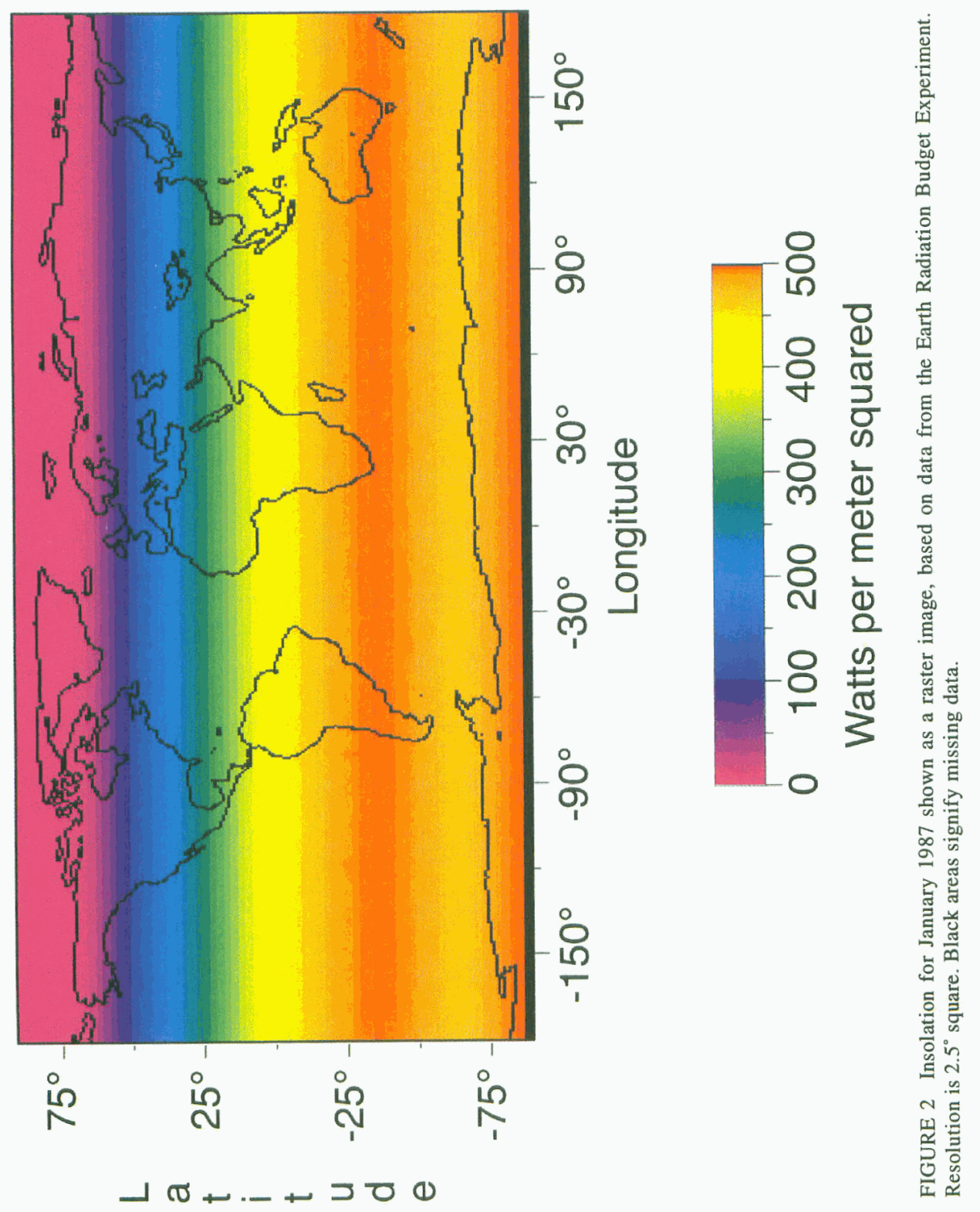

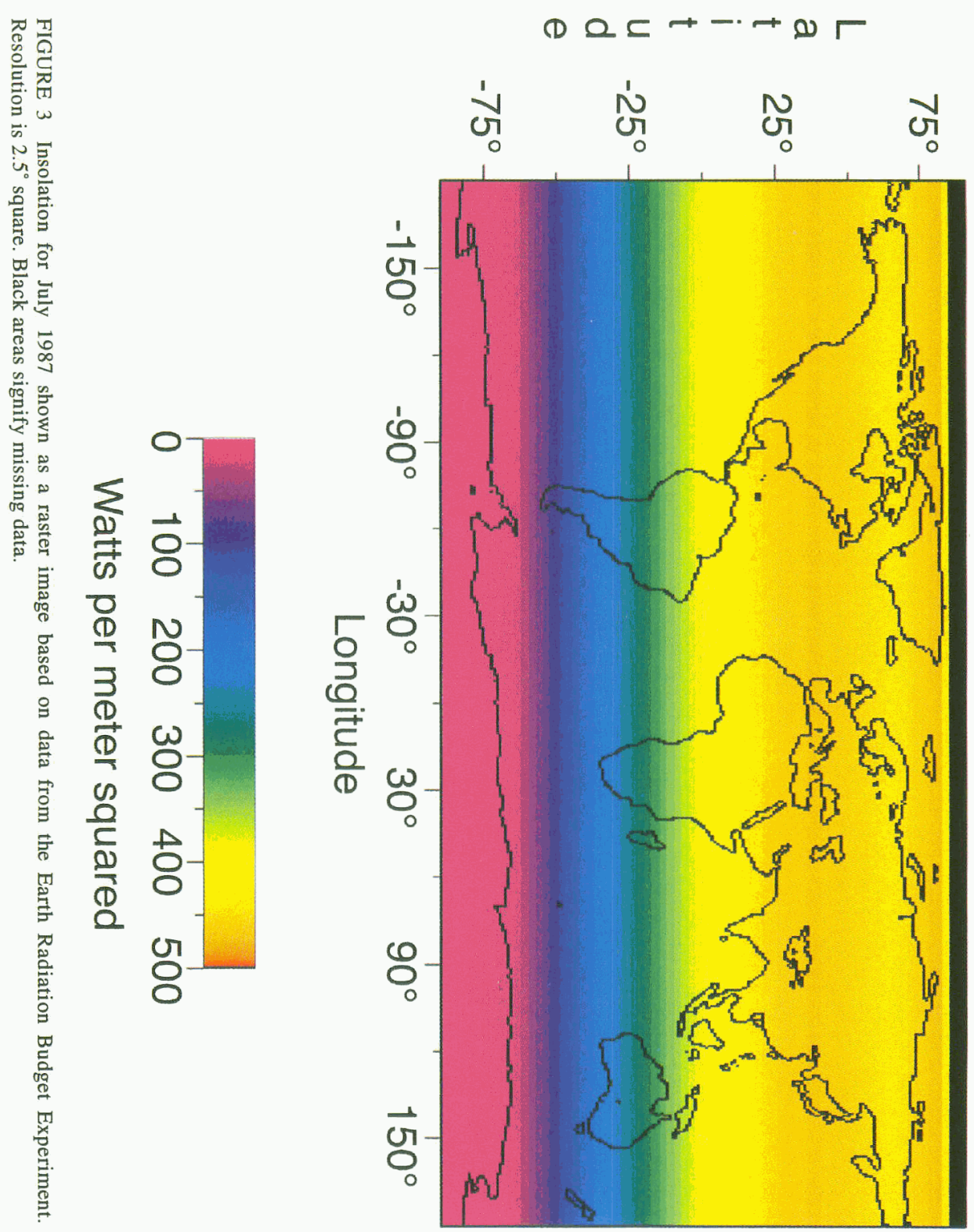

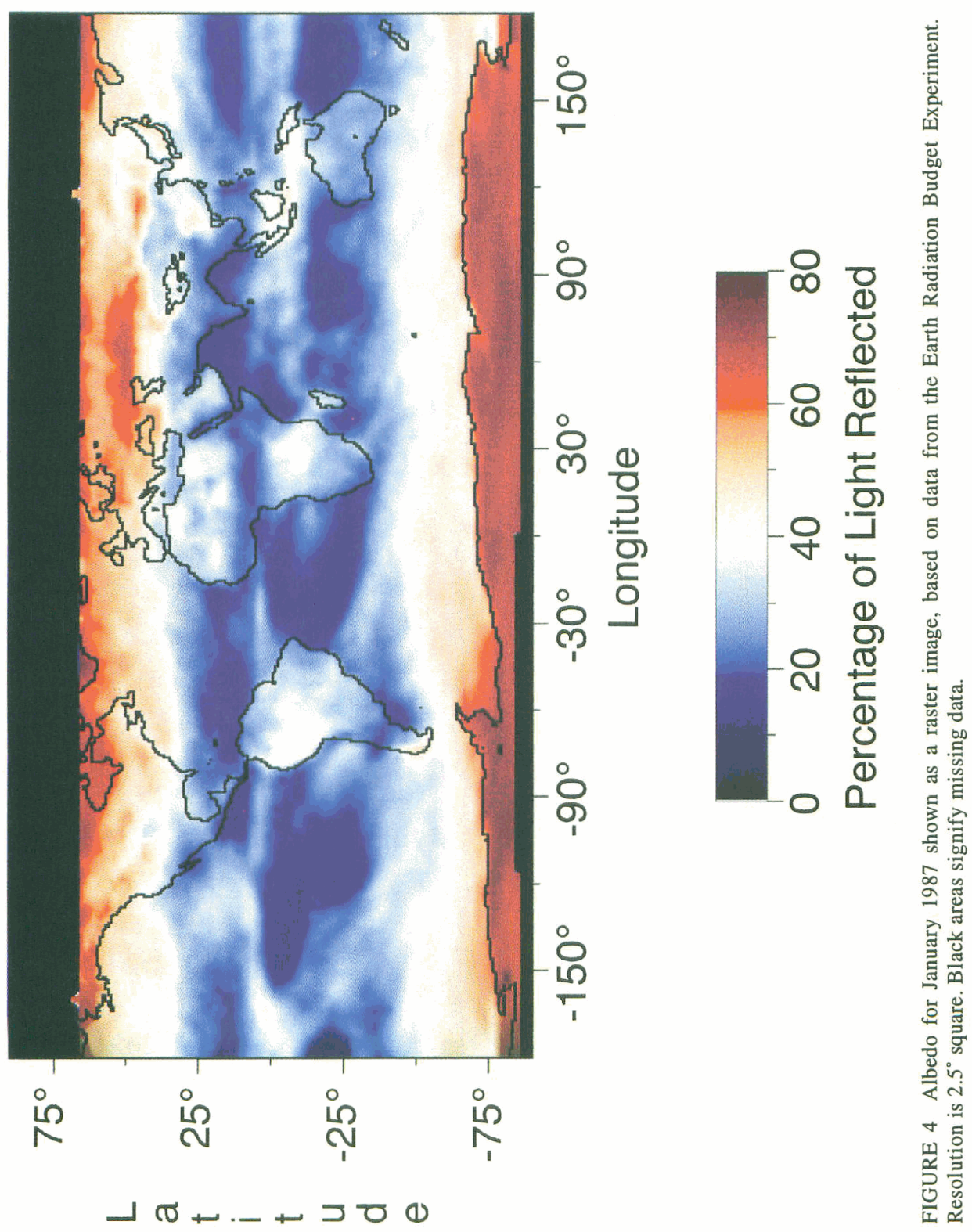

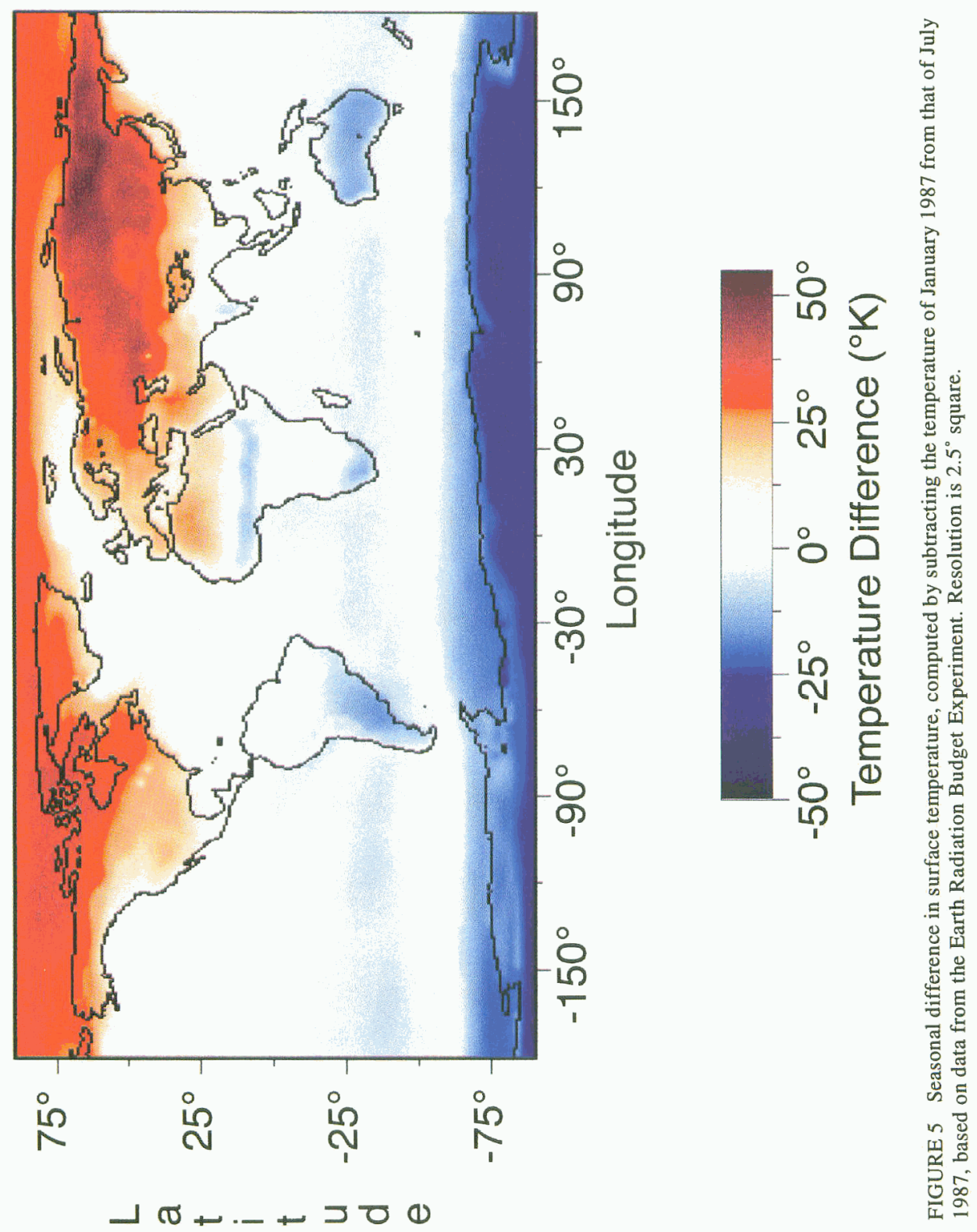
danger to them; hence, it is an issue that engages their attention. Students can inspect SciVs of the ozone hole to begin their inquiry. To acquire a robust understanding, numerous issues must be investigated: the chemical composition of ozone, the reason the ozone hole occurs in the spring over Antarctica rather than over North America, and how fast the ozone depletion is occurring.

The general point is that when inquiry begins with an issue that engages students' interest, they are more likely to energetically pursue an inquiry. Furthermore, students are interested in important issues that affect them, like the depletion of ozone. SciVs can help students begin inquiry on these issues by providing dramatic images of the phenomena they want to study, thus providing them with referents for their inquiry and discussion. As they seek to progress further, these same SciVs can provide the basis for conversations with scientists or other mentors, including more knowledgeable peers. Visual patterns in these SciVs can suggest basic lines for inquiry: Why does the ozone hole grow and shrink over the course of each year? Why is the size of the ozone hole oscillating instead of getting steadily bigger? Later, the underlying data sets can be used as the basis for experimental exploration of these questions. The questions that arise from inspecting such SciVs can quickly reach the limit of scientific knowledge. Although challenging for teachers, it is also an advantage because students engaging in inquiry in areas of current work become aware that scientific knowledge is not complete, that it has boundaries, and that it is not static but evolving as scientists continue to perform inquiry and engage in debate.

Several important issues that arise when students engage in inquiry through projects are glossed over in this account. How will the student locate the appropriate data sets? How will they find scientists willing to act as mentors? How will students understand the tacit scientific conventions such as the units employed (e.g., ozone levels are given in Dobson units). In addition, inquiry directed by the needs of a project requires topics presented outside of "textbook sequences" (e.g., students investigating ozone would need to understand the basic chemistry of ozone and how CFCs interact, but they may not have previously studied chemistry). These are difficult questions that must be addressed. In the next section, we further discuss these issues, similar issues, and some tentative directions for their solution.

\section{Empowering Students With Important Tools}

Experience using SciV can benefit students by providing them with new capabilities that could aid them in a variety of enterprises. Science is only one of the areas in which data are being transformed into virtual realities through the universal imaging abilities of computer graphics. Diverse areas are taking advantage of viewing and exploring their subject matter through computer images. Financiers view profit and loss risks within imagined 
six-dimensional spaces in which they can investigate strategic directions through navigation in visualization representations (e.g., Feiner \& Beshers, 1990). Architects inspect and refine their designs by virtually walking through their buildings before a single stone has been laid. Similarly, manufacturers test the stress levels of proposed equipment while it exists only as CAD-CAM diagrams. There is reason to believe that working with SciV and related virtual realities will be a common part of many enterprises.

\section{Laying Groundwork to Understand and Critique Scientific Issues}

SciV also offers the means to acquire a scientific literacy that is instrumental for an informed citizenry-those who do not choose to become scientists, yet wish to participate in keeping abreast of critical issues affecting their lives through, for example, medical and scientific policies. This literacy requires familiarity with rhetorical means, like SciVs, with which these issues are argued. When SciVs are used in support of project inquiry, students have an opportunity to focus their study on current scientific issues such as environmental change, depletion of the ozone, and global warming. Furthermore, with appropriate pedagogical support, students may gain expertise in the proper use of SciVs for advancing arguments and gain critical practice in discerning when SciVs are used inappropriately.

\section{Examples of SciV Within Education}

Many educators are already seeking to use SciV within their classrooms, though most have been used within postsecondary settings. To more concretely survey both the opportunities and potential problems of using SciV, we briefly review three existing programs that incorporate $\mathrm{SciV}$ as a fundamental part of their curricula. The programs we review all involve actively working with teachers, students, or both as opposed to programs that are primarily dedicated to providing access to SciVs (e.g., Sampson et al., 1994). Two of the projects we survey involve working with secondary students, and another involves working with university students.

\section{The ChemViz Project}

The ChemViz Project (Koker \& Rowe, 1993) is working with high-school chemistry teachers and students to vitalize their curriculum and pedagogy through providing access to SciVs of atomic interactions. This project is run by the National Center for Supercomputing Activities (NCSA) and is funded by the NSF. When it began in 1991, four high-school teachers were taught to 
generate the SciVs. Through the successful completion of several teacher training workshops, more than $\mathbf{3 0}$ high schools located around the country are now participating in ChemViz. Initially, the students were required to come to NCSA and program supercomputers to generate the SciVs. However, the difficulties involved in transportation and in programming the supercomputers prompted the NCSA researchers to write a "front-end" to the supercomputer software that was easy to use and that could be run over a remote connection (i.e., either Internet or modem).

The SciVs that the chemistry students request show the electron densities between atoms at various stages of bonding along with their energy levels (e.g., Koker \& Rowe, 1993). SciVs showing successive bonding stages can be combined into animations. These SciVs allow students to explore chemical bonding in depth, including the sharing of electrons, strong and weak bonds, and the effect of distance and angle on energy levels.

Informal evaluation by NCSA researchers has reported greater student motivation and increased access to difficult scientific concepts. Because students request that specific SciVs be generated, they, in effect, design their own computational chemistry experiments. This search for answers to their own questions has reportedly combined with the SciV technology to increase students' enthusiasm and has led them to spend long hours on their projects. In addition, NCSA researchers report that students are led to explore a more complex model of chemical interaction than is normally taught. A commonly taught model is the Bohr atom, in which electrons circle the atom in a fashion similar to how planets circle their sun. The more complex electron cloud model does not place an atom's electrons at a fixed distance, but describes them using probability regions. This latter model is not often used with high-school students because its understanding is often tied to other advanced material such as probability, three-dimensional geometry, waveparticle duality, and quantum mechanics. In addition, it is much harder to construct images of the electron cloud model. However, the NCSA researchers claim that by using ChemViz SciVs, which show electron clouds as atoms interact, high-school students need not be limited to the simpler Bohr model but can understand and work with the electron cloud model. This process is aided by chemistry teachers who provide guidance. The teachers take part in workshops and have continuing interactions with each other and with NCSA researchers through electronic mail.

\section{IPT Project}

The IPT project (Greenberg, 1992; Greenberg, Kolvoord, Magisos, Strom, \& Croft, 1993) reports that its innovations have produced significant changes in student motivation, particularly among underrepresented groups and at-risk students. Begun at the University of Arizona's Lunar and Planetary Laboratory in 1990, this NSF-sponsored project started with a focus on 
planetary imaging. A series of 4-week workshops were held for teachers in which a combination of lecture, hands-on training, and informal work was used to introduce the idea of teaching science through image processing. ${ }^{3}$ More than 85 teachers of Grades 5 to 12 from 16 states have now participated in these workshops and are leading image-processing activities in their science classrooms. In addition to teacher training, IPT develops curriculum and CD-ROMs containing images, though some on-site visits and continuing assistance are also available. The IPT project encourages a pedagogical style of active inquiry, styled after constructivist models of learning. They ascribe their successes, which are further detailed next, to this style of learning and to the benefits of learning through images rather than text. Images provide the means for learning, at least in part, because of the enormous amount of information they contain, as opposed to single written or spoken words.

The IPT project has not been limited to a single area of science; rather, it supplies images from a broad range of domains and has found innovative ways to perform quantitative experiments on them. Examples include measuring volcanic activity on Jupiter's moon Io, measuring lung capacity from CAT scans, and identifying seasonal and long-term changes in vegetation on a planetary scale. The analysis of images is done with NIH Image, a program developed by the National Institutes of Health (NIH) and with CD-ROMs distributed by the IPT project that contain more than 35,000 images.

Members of the IPT project have found significant effects from its curriculum of scientific image processing, particularly with underrepresented groups and at-risk students. Relying on informal assessment, the project members report that minority group members, young women, and students with "learning disabilities" were largely successful in this style of scientific inquiry. Furthermore, participating teachers reported that the negative attitudes of at-risk students were improved by engaging in supervised imageprocessing work. In addition, the members report that the teachers' perceptions of themselves changed by working with university researchers.

\footnotetext{
${ }^{3}$ The distinction between image processing and SciV requires some explanation. As suggested in the previous analogy between digital photographs and SciVs, there are substantial connections between image processing and SciV. For our purposes, the main distinction centers around whether there is access to data set or pixel values-a distinction with key ramifications on what operations can be performed. For example, the difference between two data sets can be computed only if the data set values are present. When working with images, the data have been reduced to pixels; thus, such operations cannot be meaningfully performed. Image processing differs from SciV in that it works only with the images of scientific data sets; thus, it has a reduced range of operations available. We consider this difference crucial for pedagogical purposes because students' understanding of the data and SciVs can be greatly facilitated by their performing exploratory manipulations with it. In short, SciV provides the ability to dynamically construct data sets, whereas image processing provides static images. However, for the purposes of this article, image processing is considered along with SciV because it shares the primary characteristic of pursuing scientific inquiry through interpreting images.
} 


\section{Undergraduate Geophysical Sciences Class at the University of Chicago}

Whereas the two earlier examples involved large numbers of teachers and students at middle and secondary schools, this example focuses on a single undergraduate class at a research university. The class is called Introduction to the Dynamics of Planetary Atmospheres, and it is taught by Raymond T. Pierrehumbert at the University of Chicago. ${ }^{4}$ This class uses SciV as a fundamental tool to motivate the study of climate and as a tool of inquiry. The course provides a broad introduction to the primary forces underlying Earth's climate, using the climates of Venus and Mars as contrasts.

The class time is approximately evenly split between providing qualitative descriptions of the phenomena and processes under study and deriving formal mathematical models. In particular, a series of radiative equilibrium models is introduced in which each successive model provides a more detailed picture at the expense of introducing more complexity. SciVs are used to illustrate and analyze basic climatic patterns (e.g., the slower rate of change of ocean temperature vs. land temperature). The SciVs were constructed by using Apple Macintosh computers, CD-ROM drives, and a weather data set from the National Meteorological Commission (NMC). The NMC data set provides daily measurements of temperature versus pressure and altitude versus pressure for the Northern Hemisphere over a 25-year time span. The data are particularly amenable to interpretation because they consist of measurements over a uniform latitude and longitude grid. ${ }^{5}$

Broad experiential questions are used to motivate the study of climate: What is the relation between solar radiation and the Earth's temperature? How is solar radiation distributed by the Earth's atmosphere? Why are there seasons? How can the differences between seasons be quantified? How does the temperature of the ocean relate to the temperature of land masses? These initial questions give way to questions concerning phenomena less directly experienced: What would be the climactic consequences of a comet evaporating $10 \%$ of the oceans? How is the global heat economy maintained? Are we moving toward another ice age (i.e., glaciers covering much of the continents) or toward another warm period (i.e., eras when there are no polar ice caps)? Each of these subjects is accompanied by data sets that, using SciV, serve to illustrate the phenomena under consideration.

The investigations posed by the class are divided into problem sets and data labs. The problem sets require using the models produced in class to

\footnotetext{
${ }^{4}$ The description of this class is based on personal communications with its instructor, on consulting its syllabus and handouts, and on attending its lectures during the Winter 1993 quarter.

${ }^{5}$ This grid was constructed by interpolating measurements from scattered weather stations. Hence, the data set already represents several layers of abstraction and interpretation.
} 
explain various phenomena and extending the models to handle variations in the situations considered (e.g., producing a radiative equilibrium model for Earth taking into account a "nuclear winter" scenario in which the atmosphere is surrounded by a film of black dust). The data labs draw on SciV and the climactic data sets to provide an empirical sounding board for the models constructed.

Models can also be experimentally tested using SciV. For example, a radiative transfer model can be combined with a data set of surface temperature to calculate the incoming solar radiation for comparison with measurements of insolation. Using similar techniques, "what-if" scenarios can be investigated, for example, to what extent would a thicker atmosphere (i.e., an atmosphere that absorbs more of the Earth's terrestrial radiation and therefore reemits more radiation back to Earth) produce global warming? Hence, the tools of SciV are used to test the effectiveness of models and to form predictions by combining models with data.

This course provides an example of using SciV as an integral tool for learning science. In many ways, this course exemplifies the pedagogy of cognitive apprenticeship discussed previously. Consistent with that framework, students have contact with a practicing scientist - the instructor-who does research in climatology. The students are encouraged to conduct original research, which provides them with authentic practice in the field. Although not all of these features can be easily replicated in precollege education, the course provides a working example of how SciV has been incorporated into modern day scientific apprenticeships.

\section{Summary}

The previous three examples show that the potential of SciV in education has already begun to be realized. Members of the ChemViz Project report that students are able to comprehend a more advanced model of the atom through SciVs that students request from NCSA supercomputers. Members of the IPT project report that students who traditionally have difficulties learning science are able to engage the subject matter by guided practice in image processing. The undergraduate geophysics course shows students learning SciV as one of the essential tools they will use as practicing scientists. Thus, these students, who run the gamut from at-risk students to future scientists, have benefitted from linking their science studies with the new inscriptional system of SciV. Yet, these successes have been won in spite of considerable obstacles that these projects have faced. Problems ranging from finding appropriate data sets to training teachers in the use of SciVs must be met. These problems and some tentative solutions, many of them evolving from these example projects, are surveyed next. 


\section{Potential Problems and Solutions of Using SciV in Education}

The successful incorporation of SciV into classrooms requires the resolution of complex problems. This section focuses primarily on the problems of utilizing SciV in high-school classrooms and proposes possible solutions. The discussion of problems is divided into understanding the knowledge and practices of scientists or the substance of science; the personal and social dynamics in the classroom, such as the impact of social roles and individual diversity; and the physical environment and resources.

\section{Problems Arising From Working With Data Sets}

When scientists use the inscriptional system of SciV, they draw on a large background of scientific knowledge. This knowledge is used in at least the following areas: categorizing data sets, visualizing and operating on data sets, and connecting the SciVs to scientific issues. The ramifications of students lacking this knowledge is discussed next.

Data sets. Data can influence students' inquiry by virtue of the data that are available; limitations in accuracy, resolution, or appropriateness; and the ease or difficulty in understanding the units in which the data are measured.

- The availability of data limits the domain of inquiry (e.g., if there are no data sets on black holes, then the subject cannot be studied). Hence, an important component of scientific inquiry is getting access to the data. Furthermore, the data have to be in a format that is accessible. This problem was surmounted in the IPT project by supplying CD-ROMs filled with thousands of images.

- The proper uses of a data set must be understood. Because data quality can be bad or uneven, the usefulness of data sets is limited. In general, students must understand which questions the data can help answer and how it can be utilized for these purposes. The ChemViz project and the University of Chicago climatology class handled this problem by using SciVs within the context of data labs in which many of the initial steps were prespecified.

- The units of the data must be understood. Few if any SciV tools maintain the units of the data they are manipulating - the semantics of quantities. This absence typically does not present a problem for the scientist who is guided by the semantics of domain understanding, but it does pose difficulties for students who often have had little experience with the basic conventions in which units are composed (e.g., the kilogram, meter, second system, or millibars of pressure). 
Visualizing and operating on data. The exploration and crafting of SciVs can be difficult for students because of the numerous ways numeric values can be mapped to colors and the complexities of the operations used to combine and manipulate the underlying data sets.

- The mapping from numbers to colors within a SciV can lead to confusion. In particular, the even distribution of numbers across the color palette can be misleading because equivalent numerical changes are not to be perceived as equivalent. For example, the differences found among shades of blue can seem less than those found among shades of red. Confusion can also result from palettes whose connotations conflict with the phenomena being portrayed. For example, if blue were used for warmer temperatures and red for colder ones, the student could be confused. This type of perceptual confusion can persist even if we understand its source, as has been demonstrated in work with visual illusions (Gregory, 1981).

- To understand SciVs scientists often perform operations on them. Some of these operations are simple (e.g., subtracting two data sets) and some are more mathematically complex (e.g., performing Fourier transforms). Students must have the means to perform such operations and-this is more difficult-they need to understand when to apply these operations either in the course of exploration or in forming an argument.

Connecting SciV to scientific issues. The quantities that SciVs represent are defined and designed by science. As such, students' preconceptions about scientific categories and processes will interact with their understanding of SciVs.

- Students can have preconceptions that interfere with their ability to make sense of the scientific phenomena they are examining. A rich body of research has documented student preconceptions of topics in physics, biology, and astronomy (see, e.g., reviews and monographs by Confrey, 1990; diSessa, 1993; Novak, 1987). Although one of this article's goals is to examine these issues for SciV in detail, it is important to note that these preconceptions need to be empirically discovered for each domain of study in which SciV is used.

- To make sense of the scientific phenomena portrayed by the SciVs, there is a need for a great deal of substantive knowledge. For example, when students are working with atomic interactions, as in ChemViz, they need to understand the basic composition of an atom and the ways that molecules are formed from collections of atoms. Beginning inquiry with SciVs often requires "just-in-time instruction" in contrast to well-sequenced programs of study that are fostered by following textbooks. Yet, scientific topics are often difficult to disentangle from complex preliminary topics. 


\section{Problems Arising From Social Organization}

The social organization of the classroom has an enormous impact on what the students learn. Although full consideration of this topic is beyond the scope of this article, the issues are too fundamental to neglect completely. The social organization of many science classrooms can be described as rigidly hierarchical with the teacher occupying a lead role and the students following. This organization has also been described as following an information transmission model of instruction (Pea, 1994; Pea \& Gomez, 1992). In contrast, project-enhanced science learning (Ruopp, Gal, Drayton, \& Pfister, 1993) proposes student-centered projects. This pedagogy is partially inspired by Dewey's educational philosophy but seeks to avoid the pitfalls of discovery learning by providing guidance. SciV can do little to improve secondary science education unless the social organization of the science classroom transforms in this manner. Little benefit will accrue if SciVs are merely added as new representations to existing science textbooks or as additional audio-visual aids for lectures. The potential of SciVs for education lies with an adoption of its culture of use, not just its images.

A more detailed consideration of the problems that can arise from issues of social organization and individual differences are discussed next in the categories of teachers, students, scientists, and time.

Teachers. Students' use of SciVs for project inquiry relies on teachers facilitating their access and on teachers being comfortable with using SciVs themselves.

- A class built around student projects requires the teacher to act as facilitator and manager. This role is substantially different from a teacher's traditional role, in which the teacher is the center of the classroom, both for knowledge dissemination and the initiation of activities. This shift is a difficult one for many teachers. There is reason for optimism: Members of the IPT project report that teachers attending their in-service workshops and participating in the "creative anarchy" of their research environment were helped in making this type of switch.

- Few teachers are experienced with SciVs. To guide students' investigations, teachers will need support in learning to use this new technology and in understanding how to guide their students in its use.

Students. Project inquiry relies on students having the executive skills to manage their own work. In performing these projects, students are differentially capable of utilizing resources (e.g., visually impaired students may not be able to use SciVs). 
- Students are also not accustomed to learning science through projects. The idea of taking responsibility for their learning and guiding their own inquiry can be a difficult one for them to accept (see contributions in Ruopp et al., 1993).

- Some students experience some form of color blindness ${ }^{6}$ that prevents them from using the color contrasts of SciVs. Gray-scale palettes can sometimes work as a substitute for color.

Scientists. Impeding the process of involving scientists directly in the activity of science classrooms are the difficulties of providing specific incentives for involvement, the current paucity of communication networks in schools, and the difficulty in forging common conceptual ground.

- If the practice in science classrooms is going to connect to the practice of scientists, then scientists must become involved. Yet, beyond individual charitable attitudes toward precollege education, currently there is little incentive offered to scientists for participating in scientific educational processes.

- Another factor preventing scientists' involvements in secondary science education is the difficulty of students communicating with scientists (e.g., there are few phones, modems, or Internet connections in the classroom for telecommunications; Honey \& Henriquez, 1993).

- There are many potential pitfalls in arranging for scientists to mentor high-school students. These include scientists not being able to communicate their technical knowledge and scientists making commitments that they cannot keep. Some of these difficulties can be ameliorated by the mediation of the teacher.

Time. The fragmentation of time in most schools makes in-depth investigations difficult to accomplish.

- The day of a high-school student is roughly divided into 45-min periods. Every $45 \mathrm{~min}$, students are given $5 \mathrm{~min}$ to change topics. The short length of a class is problematic for project inquiry because it does not provide enough time to pursue projects of any real complexity or depth. In addition, each period is further diminished by the needs to accommodate administrative matters, assessment procedures, and lectures, which leaves little time and even less concentration for projects.

\footnotetext{
${ }^{6}$ Eight percent of the male population and less than $1 \%$ of the female population suffer from some form of anomalous color vision. Complete dichromatism afflicts approximately $2 \%$ of the male population and a fraction of $1 \%$ of the fermale population (Meyer, 1991).
} 


\section{Problems Related to Material Resources}

Introducing SciV into secondary science classrooms also requires computers and software, networks, and data sets.

Computers and software. The use of SciVs relies on a computational infrastructure that is not present in most schools.

- Schools in the United States have acquired several million computers in the past decade. Yet, SciV often requires substantial computational power. The Chem Viz project has addressed this problem by processing the SciVs on a remote supercomputer that they connect to over a network. The specific computing resources can vary greatly with the SciVs being attempted. A relatively minimal configuration would include 4 megabytes of RAM, a floating point co-processor, a bit-mapped color display, a 40 megabyte hard disk, and a CD-ROM drive. The price for such a workstation in early 1994 was about $\$ 2,000$, whereas in 1990 it was $\$ 6,000$. In any case, there are still many schools that do not have nearly enough computers to enable their students to use SciV. In addition, the production and manipulation of SciVs can require expensive software, although excellent public domain packages are available from the NCSA and the NIH.

Networks. The synergies between high-speed communication and SciV are significant because the ability to obtain large data sets, particularly for near real-time topics (e.g., weather), depends on high bandwidth connections.

- SciV does not require that computers be connected to a network. However, a network can be used to supply some of the needs that SciV introduces, such as remote computing power and access to data sets. Unfortunately, very few high schools are currently connected to the Internet or any other high-bandwidth network (Honey \& Henriquez, 1993). Only a high-bandwidth network is sufficient because the data sets used with SciV are usually quite large, and the methods of use emphasize highly interactive usage to permit exploratory inquiry.

Data sets. Many data sets are expensive to obtain.

- As just mentioned, access to and understanding of data sets is necessary for SciV. However, data sets are often expensive. The government and many scientists do make data sets they have produced available for free or at little cost (Ross, 1994). When free data sets do exist, the use of CD-ROMs can substantially ease their dissemination. As previously mentioned, this solu- 
tion has been exploited by the IPT project, which has distributed more than 30,000 images to their associated teachers via CD-ROMs. However, other data sets are quite expensive. For example, the price for data from Landsat and Spot satellites, which take high-resolution photos of the Earth, is thousands of dollars for a single geographic region.

These problems do not have a simple solution. However, the exemplar projects provide insight into how these difficulties can be overcome and how the potential of SciV can be realized. The proposed solutions are grouped into three areas: one is infrastructure - the creation of software and accompanying data sets; one is social organization-building a community of practice that links teachers, students, and scientists; and the other is a combination of both infrastructure and social organization-connecting secondary schools to a high-speed network. These suggestions are elaborated in the next section.

Customized software and data sets. Student difficulties in understanding data sets and their SciVs can be partially solved by reifying scientists' knowledge within software environments. These software environments can provide supplementary context for SciVs, thus making explicit the knowledge that scientists use in their interpretation. To do this, the software environments need to be tied to specific data sets. This approach is consistent with the "front-end" built by NCSA researchers to aid high-school students in obtaining SciVs from their supercomputers. Software environments of this sort can provide explanatory contexts by describing what is being shown, how the data were collected, their numeric range, and their spatial and temporal extent. The contextual information does not differ appreciably from what one expects to find on a map. These cartographic standards need to be enunciated for SciVs because they so often are lacking. In addition, when data are requested, they are shown with units, and useful operations are provided on the SciV. For example, after examining a SciV of temperature, a student could request a SciV showing the extent to which it is anomalous. Research is needed to find the means to easily build software environments with these features.

The consolidation of data sets is another important area to promote educational use of SciVs. The data sets could be packaged with preexisting tools and a proposed set of open-ended projects to create a curriculum. This is the approach the IPT project team has chosen-creating CD-ROMs of images and designing curriculums. Some data sets seem particularly well suited to student use, such as national census data to allow students to understand the social makeup of their community or country; Landsat or SPOT data of the surrounding community to allow the geographic, biological, and urban characteristics to be examined; global climate and environmental data sets to understand and critique the possibilities of global change; the human ge- 
nome data set to present students with the beginnings of a map of the biological mechanisms that determine physical life; and astronomical data sets that allow students to orient themselves within our solar system, galaxy, and universe. This list is not meant to be exhaustive but to be evocative of the potential diversity that students can explore.

Building a community of practice linking students, teachers, and scientists. Transforming our schools into places in which cognitive apprenticeships can take place and in which students can successfully carry out authentic science projects requires that a community of practice be built involving students, teachers, and scientists. There are many possibilities for productive relations among these groups. Scientists can lead in-service training for teachers to introduce them to new inscriptional media like SciV. Training of this sort could lead to scientists acting as mentors and, in conjunction with science teachers, guiding students to participate in genuine scientific inquiries. Thus viewed, the population of students and teachers is an enormous untapped resource for generating scientific knowledge. Examples of this type of cooperation have occurred in the Technical Education Research Center Global Lab Project (Technical Education Research Center, 1991) and are also reported in the IPT project. In addition, the educational community can help scientists in their quest for consensus and economic backing.

A recent example of a collaboration between education and science is the National Research Education Network. The stated goals of the network were to connect public schools and to provide an "information superhighway" for data and communication links for scientific research. The combined support from these two communities helped pass the High Performance Computing Act (1991), which guarantees several billions of dollars in investment for what is now described as the National Information Infrastructure (NII). Now however, discussions of which exact services to build for the NII have led to some controversies between the two communities; the extremes are scientists hoping to connect supercomputers and educators wanting to provide network access to all public schools. Although the goals are not fundamentally incompatible, federal subsidies and network communication bandwidth are limited commodities. Hence, this example can be viewed as a possible model and as a cautionary tale for how these two communities can work together. The common ground between science and educators is elusive. More experiments are needed to see how schools can join with expert practitioners, like scientists, in the formation of communities that benefit all of their participants.

Connecting schools to a high-speed network. An important first step toward creating a community that spans students, teachers, and scientists is to forge a commonly accessible communication medium. Connecting high schools to a high-speed computer network would provide them with the 
means to interact with scientists and other mentors (Pea, 1993a). Currently, students and teachers in high schools and scientists have little means with which to contact each other and few organizing structures to promote such contact. Providing flexible asynchronous and synchronous connections can promote a new level of interaction that may result in the type of shared community previously described. Such a computer network could promote the transfer of data sets and technical data and support the social interactions that undergird successful working relationships. In addition, students could collaborate with students located at remote locations or seek guidance from them or their teachers. The network also provides a means to disseminate the results of student work, thus providing them with an audience for their work.

As our sociological account of scientific work detailed, the work of science can be described as the production of inscriptions that garner consensus and social acclaim. By linking students to one another and to the main avenues of society, students are provided with authentic motivation to engage in the scientific inquiry and the construction of scientific inscriptions. Hence, students could generate SciVs from scientific data sets received over their computer network connection and then funnel their work back over this same connection to other science students and into the general scientific community. This possibility is particularly enticing because our capacity to collect data is outstripping our ability to analyze the data. This overload is guaranteed to increase as many more satellites are sent into space as part of the Earth Observing System and as powerful new eyes and ears come online (e.g., the scanning tunneling microscope and underwater networks of sonic detectors).

\section{CONCLUSION}

The case has been made that SciV shows remarkable potential to help students learn science. As an extraordinarily plastic medium, SciV affords the construction of provocative images that can resemble physical phenomena and serve as the basis for the construction, debate, and negotiation of meaning that stands at the heart of the process of education. However, research is needed to understand how students come to be able to read and understand SciVs and how this process can be facilitated by material and social supports. When students succeed in appropriating the inscriptions of SciV, grounds are laid to connect the education of students with the practices of scientists. This connection holds promise for students' studies to achieve authenticity or consonance with the practices and goals of science. Yet, considerable research is needed to forge a clear plan for how scientists and students can best interact.

It is here, in the interface between scientist and student, that the science educator can play the profoundly useful role of diplomat and interpreter. 
This role is especially challenging, because the practices and goals of science and education have drifted apart, leaving each stranded in separate communities. Yet, the resulting lack of connection is not to the benefit of either one. The integration of science and education will require the searching gaze of ethnography to understand the starting goals and practices of each community, brave educational experiments that seek to act on these understandings, and rigorous assessment to interpret the results. Although starting points in this process are often elusive, the ground between learners' preconceptions of the physical world and scientists' tools or inscriptional mediums like SciV can provide an advantageous place to begin.

\section{ACKNOWLEDGMENTS}

We are grateful for research support of the CoVis Project by the National Science Foundation Grant No. MDR-9253462; by Apple Computer, Inc., External Research; by Sun Microsystems; and by our industrial partners Ameritech and Bellcore. We also thank our colleagues from the CoVis Project and community of users for extended discussions of these issues and continual, useful feedback on design, rationale, and pedagogical issues. Special thanks to climatology professor Raymond T. Pierrehumbert for his initiation and support of our entries into the wide world of climate data sets and tools. In addition, we are indebted to our two reviewers at The Journal of the Learning Sciences.

\section{REFERENCES}

Barkstrom, B. R. (1984). The earth radiation budget experiment (ERBE) data sets [Machinereadable data file]. Hampton, VA: Atmospheric Sciences Division NASA/Langley Research Center (Producer). Greenbelt, MD: NASA Climate Data System, Distributed Active Archive Center (Distributor).

Becker, R. A., \& Cleveland, W. S. (1991, July-August). Take a broader view of scientific visualization. Pixel, pp. 42-44.

Brodie, K. W., Carpenter, L. A., Earnshaw, R. A., Gallop, J. R., Hubbold, R. J., Mumford, A. M., Osland, C. D., \& Quarendon P. (1992). Scientific visualization. Berlin, Germany: SpringerVerlag.

Brown, J. S., Collins A., \& Duguid, P. (1989). Situated cognition and the culture of learning. Educational Researcher, 18, 32-42.

Cohen, D. K. (1988). Educational technology and school organization. In R. S. Nickerson \& P. P. Zodhiates (Eds.), Technology in education: Looking toward 2020 (pp. 231-264). Hillsdale, NJ: Lawrence Erlbaum Associates, Inc.

Confrey, J. (1990). A review of the research on student conceptions in mathematics, science, and programming. In C. Cazden (Ed.), Review of research in education (Vol. 16, pp. 3-56). Washington, DC: American Educational Research Association.

Cuban, L. (1986). Teachers and machines: The classroom use of technology since 1920. New York: Teachers College Press.

diSessa, A. (1993). Toward an epistemology of physics. Cognition and Instruction, 10, 105225 . 
Domenico, B., Bates, S., \& Fulker, D. (1994, January). Unidata internet data distribution (IDD). In D. R. Smith \& L. Bastiaans (Chairs), Proceedings of the Third Symposium on Education of the American Meteorological Society. Nashville, TN.

Elvins, T. T. (1992). A survey of algorithms for volume visualization. Computer Graphics, 26, 194-201.

Feiner, S., \& Beshers, C. (1990). Visualizing $n$-dimensional virtual worlds with $n$-Vision. Proceedings of the 1990 Symposium on Interactive 3D Graphics (pp. 37-38). Snowbird, UT.

Greenberg, R. (1992, November). Scanning the images of science. The Science Teacher, pp. 14-18.

Greenberg, R., Kolvoord, R. A., Magisos, M., Strom, R. G., \& Croft, S. (1993). Image processing for teaching. Journal of Science Education and Technology, 2, 469-480.

Gregory, R. L. (1981). Mind in science. New York: Cambridge University Press.

Griffin, P., \& Cole. M. (1987). Computers' impact on the context of instruction. In P. Griffin \& M. Cole (Eds.), Contextual factors in education (pp. 43-62). Madison: Wisconsin Center for Education Research.

Hall, S. S. (1992). Mapping the next millennium. New York: Vintage.

Hawkins, J., \& Pea, R. D. (1987). Tools for bridging everyday and scientific thinking. Journal for Research in Science Teaching, 24, 291-307.

High Performance Computing Act of 1991, Pub. L. No. 102-194.

Honey, M., \& Henriquez, A. (1993). Telecommunications and $K-12$ educators: Findings from a national survey. New York: Bank Street College of Education.

Knorr-Cetina, K. D. (1981). The manufacture of knowledge. New York: Pergamon.

Koker, T., \& Rowe, B. (1993). ChemViz: An NSF sponsored program to bring high performance computing to America's high schools [Machine-readable data file], Urbana-Champaign: University of Illinois, National Center for Supercomputing Applications ChemViz Project (Producer). Urbana-Champaign: University of Illinois, National Center for Supercomputing Applications (Distributor).

Latour, B. (1988). Drawing things together. In M. Lynch \& S. Woolgar (Eds.), Representation in scientific practice (pp. 19-68). Cambridge, MA: MIT Press.

Latour, B., \& Woolgar, S. (1986). Laboratory life: The construction of scientific facts. Princeton, NJ: Princeton University Press.

Lave, J., \& Wenger, E. (1991). Situated learning: Legitimate peripheral participation. Cambridge, England: Cambridge University Press.

Linn, M. C., diSessa, A., Pea, R. D., \& Songer, N. B. (in press). Can research on science learning and instruction inform standards for science education? Journal of Science Education and Technology.

Linn, M. C., Songer, N. B., \& Eylon, B. (in press). Shifts and convergences in science learning and instruction. In D. Berliner \& R. Calfee (Eds.), Handbook of educational psychology.

Lynch, M., \& Woolgar, S. (1988). Introduction: Sociological orientations to representational practice in science. In M. Lynch \& S. Woolgar (Eds.). Representation in scientific practice (pp. 1-18). Cambridge, MA: MIT Press.

McCormick, B. H., DeFanti, T. A., \& Brown, M. D. (Eds.). (1987). Visualization in scientific computing. Computer Graphics, 2l(6).

Meyer, G. W. (1991). Color vs. black-and-white in visualization. In G. M. Nielson \& L. Rosenblum (Eds.), Proceedings of Visualization '91 (pp. 336-339). Los Alamitos, CA: IEEE Computer Society Press.

National Competitiveness Act of 1993, 473 S. $\$ 1105$.

Novak, J. D. (Ed.). (1987). Proceedings of the second international seminar on misconceptions and teaching strategies in science and mathematics. Ithaca, NY: Cornell University.

Office of Science and Technology Policy. (1991, February). Grand challenges: High performance computing and communications: The FY 1992 U.S. research and development program. (A report by the Committee on Physical, Mathematical, and Engineering Sciences, 
Federal Coordinating Council for Science, Engineering, and Technology.) Washington, DC: Executive Office of the President.

Pea, R. D. (1992). Augmenting the discourse of learning with computer-based learning environments. In E. de Corte, M. Linn, H. Mandl, \& L. Verschaffel (Eds.), Computerbased learning environments and problem-solving (pp. 313-343). New York: SpringerVerlag.

Pea, R. D. (1993a). The Collaborative Visualization Project. Communications of the ACM, $36(5), 60-63$.

Pea, R. D. (1993b). Practices of distributed intelligence and designs for education. In G. Salomon (Ed.), Distributed cognition (pp. 47-87). New York: Cambridge University Press.

Pea, R. D. (1994). Seeing what we build together: Distributed multimedia learning environments for transformative communications. The Journal of the Learning Sciences, 3, 285-299.

Pea, R., \& Gomez, L. (1992). Distributed multimedia learning environments: Why and how? Interactive Learning Environments, 2, 73-109.

Postman, N. (1992). Technopoly: The surrender of culture to technology. New York: Knopf.

Ross, T. F. (1994, January). NCDC's CD-ROM products and on-line services-A useful tool for educators preparing students for the 21 st century. In D. R. Smith \& L. Bastiaans (Chairs), Proceedings of the Third Symposium on Education of the American Meteorological Society. Nashville, TN.

Ruopp, R., Gal, S., Drayton, B., \& Pfister, M. (Eds.). (1993). LabNet: Toward a community of practice. Hillsdale, NJ: Lawrence Erlbaum Associates, Inc.

Sampson, P., Sternberg, A., Ferguson, J., Kamprath, M., Masters, J., Hay, K., Monan, M., \& Mullen, T. (1994, January). Blue-skies: A new interactive teaching tool for K-12 education. In D. R. Smith \& L. Bastiaans (Chairs), Proceedings of the Third Symposium on Education of the American Meteorological Society. Nashville, TN.

Szlichcinski, K. P. (1979). Diagrams and illustrations as aids to problem solving. Instructional Science, 8, 253-274.

Technical Education Research Center. (1991). Global laboratory notebook. Cambridge, MA: Author.

Toulmin, S. (1953). The philosophy of science. New York: Harper \& Row.

Watson, J. D. (1968). The double helix. New York: Atheneum.

Wolff, R., \& Yaeger, L. (1993). Visualization of natural phenomena [includes CD-ROM]. New York: Springer-Verlag. 